\section{Over-the-scope clipping in recurrent colonic diverticular bleeding}
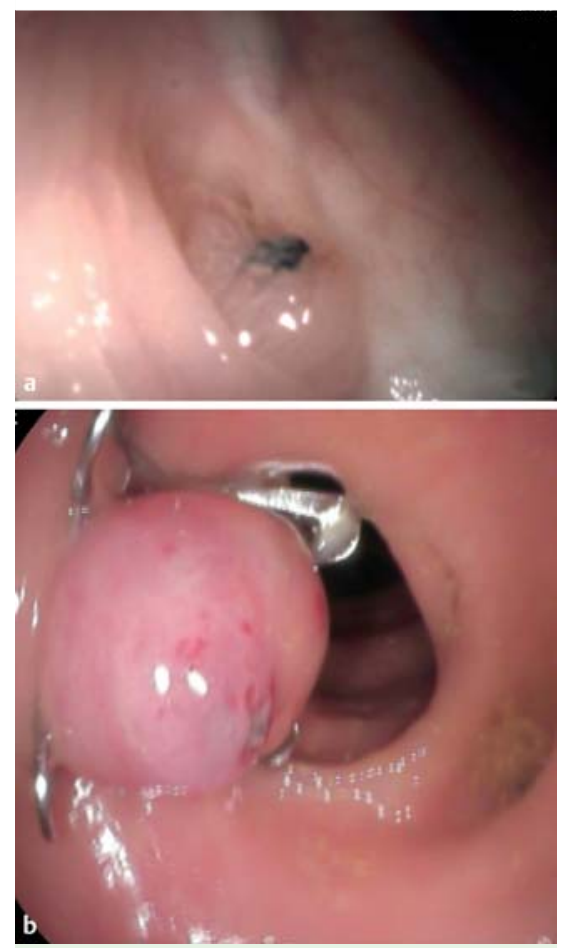

Fig. 1 Endoscopic views showing: a the bleeding source, a visible vessel on the neck of a diverticulum immediately above the colorectal anastomosis; b complete mechanical closure of the diverticular bleeding source after deployment of an over-the-scope clip (OTSC).

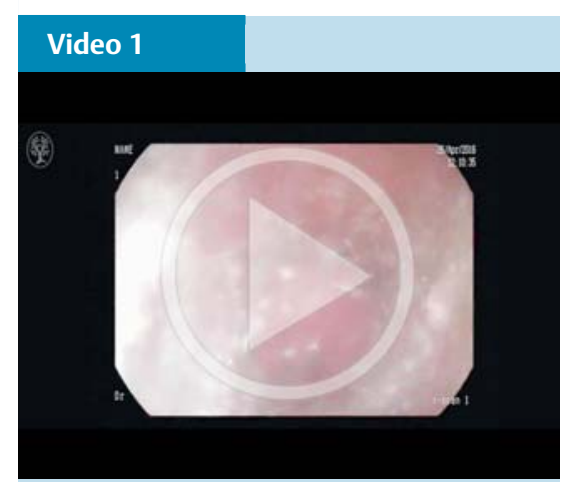

Endoscopic view of the bleeding source on the neck of a diverticulum being treated with an over-the-scope clip (OTSC) and appearance at repeat endoscopy 3 months later.
Colonic diverticula are the most frequent cause of lower gastrointestinal (GI) bleeding in adults. Nonetheless, the identification of a definite bleeding source is endoscopically challenging and diagnosis is usually presumptive. Once a source has been precisely identified, various endoscopic hemostatic methods can be used, including clipping, endoscopic band ligation and epinephrine injection [1,2]. However, use of an over-the-scope clip (OTSC) has not yet been described.

Here, we report the case of a 76-year-old man with a history of recurrent bleeding from diffuse and severe colonic diverticulosis that had been previously treated with left-sided colectomy. In the 2 years following this, he experienced four new episodes of severe diverticular bleeding from the residual colon with spontaneous resolution, thereby providing an indication for total colectomy.

When a colonoscopy was performed after the last of his admissions, for a 3-day history of persistent lower GI bleeding, a visible vessel with pigmented hematin was seen on the neck of a diverticulum just above the colorectal anastomosis ( 0 Fig. 1 a). Notably, no additional bleeding source was identified throughout the remainder of the colon and an upper GI endoscopy was negative for bleeding.

Given the need to provide long-term hemostatic treatment and the presence of peri-anastomotic stiffness, endoscopic band ligation was not feasible, so we therefore decided to place an OTSC with a 6-mm depth and pointed teeth (Ovesco Endoscopy, Tübingen, Germany) [3]. The OTSC was successfully deployed at the first attempt, more than half of the volume of the clear distal cap having been filled with tissue, followed by suctioning and inversion of the bleeding diverticulum. Particular attention was paid to placing the vessel that was bleeding into the center of the clip, thereby allowing

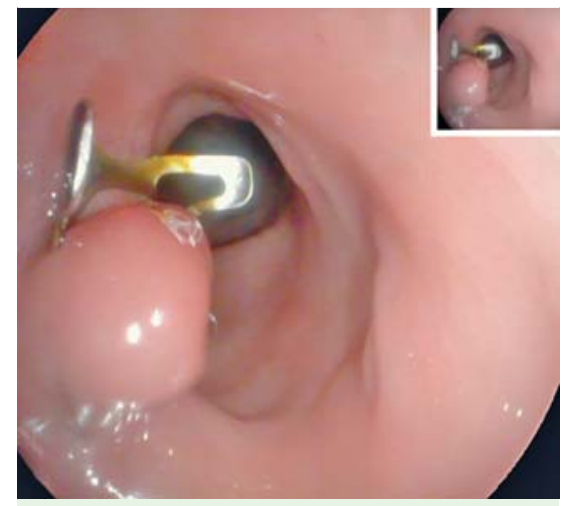

Fig. 2 Endoscopic appearance 3 months after the procedure with no further evidence of the bleeding source.

for immediate and complete mechanical closure $(\bullet$ Fig. $1 \mathbf{b}$; $\bullet$ Video 1$)$.

No immediate complications occurred and the patient was discharged 4 days later. There were no short-term complications and no recurrent bleeding had occurred after 3 months of follow-up ( $\bullet$ Fig. 2 ).

This report illustrates for the first time the feasibility and efficacy of hemostasis by endoscopic clipping with the OTSC system for diverticular bleeding. Thanks to this simple and immediate endoscopic approach, our patient was able to avoid an aggressive surgical strategy, which would have been burdened by considerable risks and functional consequences.

\section{Endoscopy_UCTN_Code_TTT_1AQ_2AZ}

\section{Competing interests: None}

Paola Soriani ${ }^{1}$, Gian Eugenio Tontini', Sara Vavassori ${ }^{1}$, Helmut Neumann ${ }^{2}$, Luca Pastorelli ${ }^{1,3}$, Maurizio Vecchi ${ }^{1,3}$, Pavlos Lagoussis ${ }^{4}$

${ }^{1}$ Gastroenterology and Digestive Endoscopy Unit, IRCCS Policlinico San Donato, San Donato Milanese, Italy

${ }^{2}$ Department of Medicine I, University of Erlangen-Nuremberg, Erlangen, Germany

${ }^{3}$ Department of Biomedical Sciences for Health, University of Milan, Milan, Italy

${ }^{4}$ Division of General Surgery I, IRCCS Policlinico San Donato, San Donato Milanese, Italy 


\section{References}

1 Yamada A, Niikura R, Yoshida $S$ et al. Endoscopic management of colonic diverticular bleeding. Dig Endosc 2015; 27: 720 - 725

2 Shimamura $Y$, Ishii $N$, Omata $F$ et al. Endoscopic band ligation for colonic diverticular bleeding: possibility of standardization. Endosc Int Open 2016; 4: E233-E237

3 Ghassemi KA, Jensen DM. Evolving techniques for gastrointestinal endoscopic hemostasis treatment. Expert Rev Gastroenterol Hepatol 2016; 10: 615-623

\section{Bibliography}

DOI http://dx.doi.org/

10.1055/s-0042-115942

Endoscopy 2016; 48: E306-E307

(c) Georg Thieme Verlag KG

Stuttgart · New York

ISSN 0013-726X
Corresponding author

Paola Soriani, MD

Gastroenterology Digestive Endoscopy Unit IRCCS Policlinico San Donato

Via Morandi 30

San Donato Milanese

Milano 20097

Italy

Fax: +39-02-52774655

paola.soriani@gmail.com 